\title{
Transfer of Sarcina Goodsir from the Family Micrococcaceae Pribram to the Family Peptococcaceae Rogosa
}

\author{
MORRISON ROGOSA \\ Laboratory of Microbiology, National Institute of Dental Research, \\ National Institutes of Health, Bethesda, Maryland 20014
}

\begin{abstract}
Peptococcus Kluyver and van Niel, Peptostreptococcus Kluyver and van Niel, and Ruminococcus Sijpestein have recently been placed in the family Peptococcaceae Rogosa. Because Sarcina Goodsir also comprises gram-positive, anaerobic cocci whose general metabolism and other characteristics are consistent with the characteristics of Peptococcaceae, it is herein proposed that the genus Sarcina Goodsir be transferred from Micrococcaceae Pribram to Peptococcaceae Rogosa.
\end{abstract}

The genera Peptococcus Kluyver and van Niel (4), Peptostreptococcus Kluyver and van Niel (4), and Ruminococcus Sijpestein (A. K. Sijpestein, Ph.D. thesis, Univ. of Leiden, The Netherlands, 1948) recently have been included in a new family, Peptococcaceae Rogosa (6). The descriptions of these gram-positive, anaerobic cocci have also been updated (6).

On further reflection, it appears that there is nothing in the description of Peptococcaceae (6) which is inconsistent with the generic concept of Sarcina Goodsir as propounded in modern terms by Canale-Parola et al. (2) and Canale-Parola (1). Although the cell arrangement of Sarcina is in cubical packets, the description of Peptococcaceae is comprehensive enough to permit this. The general metabolic characteristics of Sarcina, particularly glucose fermentation with major production of $\mathrm{CO}_{2}$, $\mathrm{H}_{2}$, certain lower fatty acids, variable (as to species) production of ethanol, and lack of lactic acid as a major product are also consistent with the description of Peptococcaceae (6). Other definitively known characteristics of Sarcina, including the ability to grow at extremely acid $(p \mathrm{H} 1)$ and alkaline $(p \mathrm{H} 9.8)$ reactions, are permissible as Peptococcaceae was described (6).

The packet-forming cocci capable of aerobic growth are phylogenetically distant from those which are strictly anaerobic. For example, the percentage of moles of guanine plus cytosine in the deoxyribonucleic acid of the aerobic species $S$. lutea is 73.5 , whereas the deoxyribonucleic acid from $S$. maxima and $S$. ventriculi, both anaerobes, give values of 28.6 and 30.6 , respectively (2). Canale-Parola et al. (2) have ably summarized the evidence for including only the strictly anaerobic sugar-fermenting species $(S$. ventriculi and $S$. maxima) in the genus Sarcina. Also, Sarcina was first used by Goodsir in 1842 to name $S$. ventriculi, one of the two species of anaerobic, sugar-fermenting, packet-forming cocci presently recognized.

From the foregoing, it seems appropriate to include Sarcina Goodsir in the same family as certain other genera of gram-positive, anaerobic cocci. Therefore, it is here proposed to transfer Sarcina Goodsir from Micrococcaceae Pribram (5) to Peptococcaceae Rogosa. Thus, the genera of Peptococcaceae Rogosa are: (i) Peptococcus Kluyver and van Niel, 1936 [type species $P$. niger (Hall) Kluyver and van Niel]; (ii) Peptostreptococcus Kluyver and van Niel, 1936 [type species. $P$. anaerobius (Krönig) Kluyver and van Niel]; (iii) Ruminococcus Sijpestein, 1948 (type species, $R$. flavefaciens Sijpestein); and (iv) Sarcina Goodsir, 1842 (type species, $S$. ventriculi Goodsir).

Peptococcaceae Rogosa, Peptococcus Kluyver and van Niel, Peptostreptococcus Kluyver and van Niel, and Ruminococcus Sijpestein have been described recently in contemporary terms (6); Sarcina Goodsir has been described in updated terms by CanaleParola et al. (2) and Canale-Parola (1). The general differentiating characteristics of the genera of Peptococcaceae are presented in Table 1. 


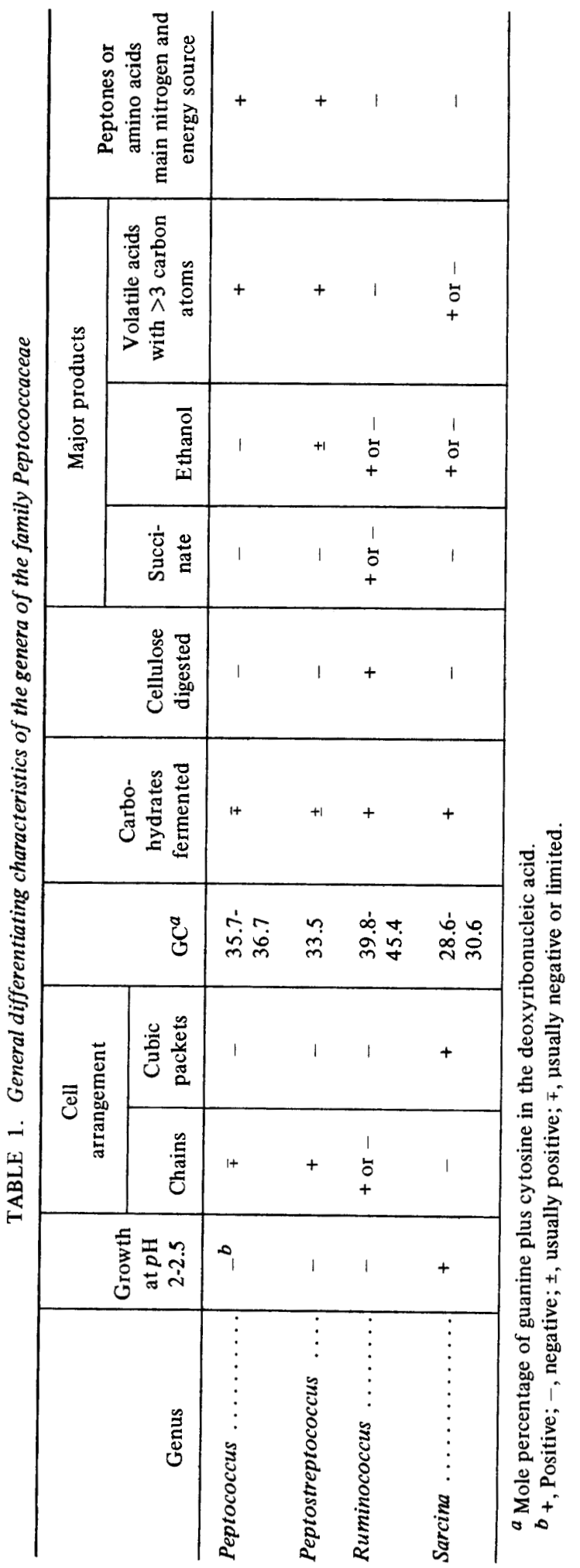




\section{LITERATURE CITED}

1. Canale-Parola, E. 1970. Biology of the sugarfermenting sarcinae. Bacteriol. Rev. 34:82-97.

2. Canale-Parola, E., M. Mandel, and D. G. Kupfer. 1967. The classification of sarcinae. Arch. Mikrobiol. 58:30-34.

3. Goodsir, J. 1842. History of a case in which a fluid periodically ejected from the stomach contained vegetable organisms of an undescribed form. With a chemical analysis of the fluid, by George Wilson. Edinburgh Med. Surg. J. 57:430-443.
4. Kluyver, A. J., and C. B. van Niel. 1936. Prospects for a natural system of classification of bacteria. Zentrabl. Bakteriol. Parisitenk. Infektionskr. Hyg. Abt. II 94:369-403.

5. Pribram, E. 1929. A contribution to the classification of microorganisms. J. Bacteriol. 18:361-394.

6. Rogosa, M. 1971. Peptococcaceae, a new family to include the gram-positive, anaerobic cocci of the genera Peptococcus, Peptostreptococcus, and Ruminococcus. Int. J. Syst. Bacteriol. 21:234-237. 